\title{
Management of Anaplastic Thyroid Carcinoma: the Fruits from the ATC Research Consortium of Japan
}

\author{
Iwao Sugitani ${ }^{1}$, Naoyoshi Onoda ${ }^{2}$, Ken-ichi Ito $^{3}$ and Shinichi Suzuki ${ }^{4}$ \\ ${ }^{1}$ Department of Endocrine Surgery, Nippon Medical School Graduate School of Medicine, Tokyo, Japan \\ ${ }^{2}$ Department of Surgical Oncology, Osaka City University Graduate School of Medicine, Osaka, Japan \\ ${ }^{3}$ Division of Breast, Endocrine and Respiratory Surgery, Department of Surgery (II), Shinshu University School of Medicine, Nagano, Japan \\ ${ }^{4}$ Department of Thyroid and Endocrinology, Fukushima Medical University School of Medicine, Fukushima, Japan
}

\begin{abstract}
Anaplastic thyroid carcinoma (ATC) accounts for only 1 to $2 \%$ of all thyroid carcinomas, but it is one of the most lethal neoplasms in humans. To obtain further insights into this "orphan disease," we have established the ATC Research Consortium of Japan (ATCCJ) in 2009. It represents a multicenter registry for ATC that have been treated in Japan. To date, 67 institutions have taken part in the collaborative research system and over 1,200 cases have been accumulated in its database. Using this big data, several retrospective studies were carried out to evaluate 1) prognostic factors to determine initial treatment policy, 2) significance of extended radical surgery for Stage IVB cases, 3) characteristics of ATC incidentally found on pathological examination and 4) pathological features of ATC with long-term survival. Moreover, the ATCCJ has conducted an investigator-initiated, nationwide, prospective clinical trial since 2012; namely, the feasibility, safety and efficacy study of weekly paclitaxel administration for patients with ATC (UMIN: 000008574). Revised Japanese guidelines for treatment of thyroid tumors are going to adopt the recommendations from the results of this research. Since 2016, the ATCCJ has started the phase II study assessing the efficacy and safety of lenvatinib, a newly developed tyrosine kinase inhibitor for ATC (UMIN: 000020773). Our nationwide clinical trial network will strengthen the activity to recruit orphan disease patients and may discover new strategies to conquer this dismal malignancy in the near future. (J Nippon Med Sch 2018; 85: 18-27)
\end{abstract}

Key words: anaplastic thyroid carcinoma, orphan disease, nationwide multi-institutional research consortium, investigator-initiated clinical trials, guidelines

\section{Introduction}

Anaplastic thyroid carcinoma (ATC) is uncommon and represents only $1-2 \%$ of all thyroid carcinomas, but accounts for $14-39 \%$ of thyroid cancer deaths ${ }^{1,2}$. ATC is one of the most lethal neoplasms in humans, with a dismal prognosis as $5-20 \%$ of one-year survival. The aggressive nature and rarity of this disease make it difficult to improve the quality of evidence with regard to ATC. So far, most existing knowledge about ATC is derived from single-institution studies with limited numbers of cohorts and short-term follow-up. To obtain further insights into this "orphan disease", we established the ATC Research Consortium of Japan (ATCCJ) in January 2009. Objectives of the multi-institutional collaborative study group were (1) to create a multi-center database that accumulates all information for ATC available in Japan, (2) to carry out retrospective studies that analyze the big data, (3) to conduct prospective studies including clinical trials to evaluate controversial or novel strategies, and (4) to revise guidelines based on new evidence. Our ultimate goal is to improve the treatment outcome of patients with ATC.

To date, 67 institutions have taken part in the ATCCJ and over 1,200 cases have been registered in its database. The ATCCJ has reported several retrospective studies using the database to upgrade evidence about the management of ATC. In addition, we have conducted

Correspondence to Iwao Sugitani, Department of Endocrine Surgery, Nippon Medical School Graduate School of Medicine, 11-5 Sendagi, Bunkyo-ku, Tokyo 113-8603, Japan

E-mail: isugitani@nms.ac.jp

Journal Website (http://www2.nms.ac.jp/jnms/) 
Cause-specific survival rate (\%)

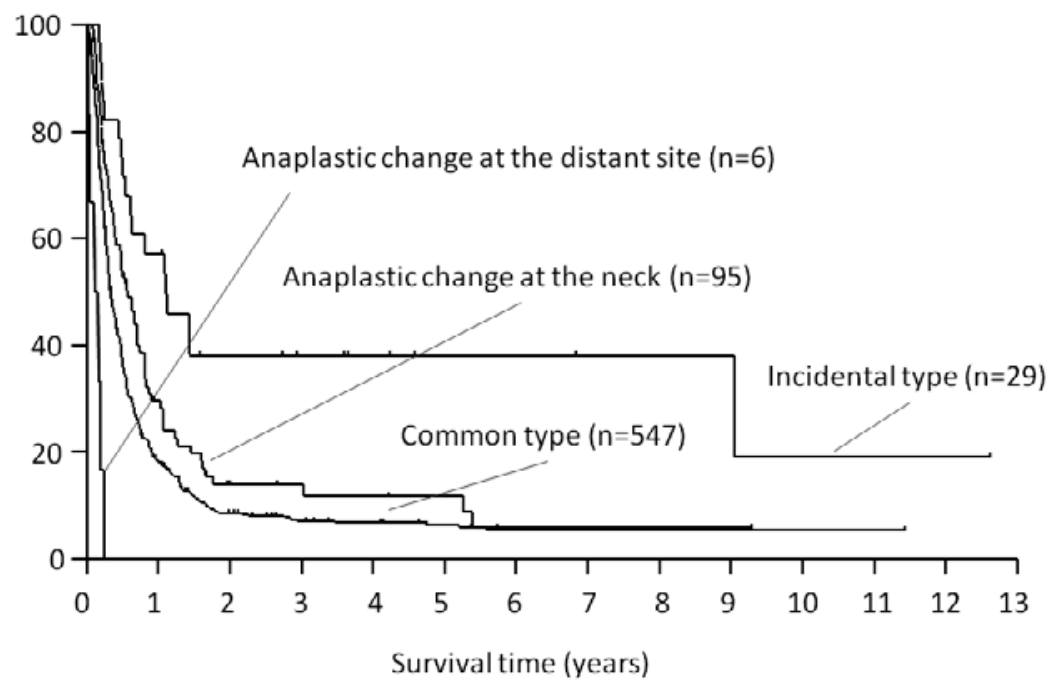

Fig. 1 Cause-specific survival curves for each clinical type of anaplastic thyroid carcinoma

investigator-initiated, nationwide, prospective clinical trials. Revised Japanese guidelines for the treatment of thyroid tumors are going to adopt these recommendations. In this review, we introduce our achievements and future directions to conquer this dismal malignancy.

\section{Overall Treatment Outcomes for ATC}

In 2012, we reported the treatment outcomes of a cohort of 677 ATC patients treated at 38 institutions during the 14-year period between 1995 and 2008 ${ }^{3}$. Thirty-three patients for whom diagnosis was not achieved by pathological or cytological examination (i.e., only by clinical presentation) were excluded from the study. Clinical varieties of ATC were classified into the 4 following types: common type $(n=547)$; incidental type $(n=29)$; anaplastic transformation at the neck lymph node(s) $(\mathrm{n}=95)$; and at a distant site $(n=6)$. Incidental ATC was largely differentiated thyroid carcinoma (DTC) accompanied by a minute $(1-2 \mathrm{~cm})$ region of ATC, and anaplastic transformation type was ATC at a site other than the thyroid gland, which was associated with previous or concomitant DTC at the thyroid.

Survival curves for each clinical type of ATC are shown in Figure 1. As for 547 patients with common type ATC, there were 208 males and 339 females (1 : 1.6) and mean age was $68.7 \pm 11.0$ years (range: 28 to 100). Their mean survival time (MST) was 113 days (range: 1 to 4,174) and 6-months and 1-year cause-specific survival (CSS) rate were $36 \%$ and $18 \%$, respectively. Patients with incidental ATC showed significantly better outcomes than other types $(\mathrm{p}<.0001)$. Survival for anaplastic transformation at the neck lymph node(s) was also better than that for common type $(p=.0082)$. Anaplastic change at a distant site had the worst prognosis $(\mathrm{p}<.0001$ compared to common type).

\section{Prognostic Factors for Common Type ATC}

Some ATC patients survive a fairly long time after aggressive multimodal treatment combining radical surgical resection, sufficient dose of external-beam radiation therapy (RTX) and some kind of chemotherapy (CTX); however, radical treatment may worsen the quality of life (QOL) and occasionally even shorten survival. Selecting patients who will benefit from either aggressive therapy or only supportive care is important in the management of ATC. In 2001, Sugitani et al. devised a "prognostic index" (PI) based on the number of 4 unfavorable prognostic factors present in the patient with $\mathrm{ATC}^{4}$. The factors were (1) acute symptoms (duration of severe complaints such as dysphonia, dysphagia, dyspnea, and rapid growth of the tumor $<1$ month); (2) leukocytosis (white blood cell count $\geq 10,000 / \mathrm{mm}^{3}$ ); (3) tumor size $>5 \mathrm{~cm}$, and (4) distant metastasis. In the original study, patients with $\mathrm{PI} \leq 1$ showed a $62 \%$ survival rate at 6 months, whereas no patients with $\mathrm{PI} \geq 3$ survived longer than 6 months. All patients with $\mathrm{PI}=4$ died within 3 months. They recommended attempting multimodal treatment for patients with $P I \leq 1$, while avoiding aggressive treatment to maintain QOL for patients with $\mathrm{PI} \geq 3$. Adopting the PI prospectively, Orita et al. evaluated the validity of PI and 
the therapeutic strategy in 2012. Compared to the historical control, prospective cases with $\mathrm{PI} \leq 1$ underwent multimodal treatment more frequently and showed significantly better survival. For $\mathrm{PI} \geq 3$ patients, survival rates were equally dismal; however, the numbers of patients who underwent tracheostomy or died from local disease were significantly lower compared to the previous cases. They concluded that PI was a useful tool in predicting the prognosis and choosing the proper therapeutic strategy for individual patients ${ }^{5}$.

Using the ATCCJ database, multivariate analysis of prognostic factors was carried out for survival of the 547 patients with common-type ATC. The $\mathrm{T}$ classification (T4a: tumor limited to the thyroid; T4b: tumor extending beyond the thyroid capsule) and staging (IVA: T4a Any N M0; IVB: T4b Any N M0; IVC: Any T Any N M1) were

Table 1 Multivariate analysis of prognostic factors in patients with common-type ATC

\begin{tabular}{lccc}
\hline \multicolumn{1}{c}{ Risk factors } & $\begin{array}{c}\text { Hazard } \\
\text { ratio }\end{array}$ & $\begin{array}{c}95 \% \text { confidence } \\
\text { interval }\end{array}$ & $\mathrm{p}$ \\
\hline Age $\geq 70$ years & 1.28 & $1.04-1.58$ & 0.020 \\
Male & 1.09 & $0.88-1.36$ & 0.42 \\
Acute symptoms & 1.34 & $1.06-1.69$ & 0.014 \\
Leukocytosis & 1.48 & $1.18-1.87$ & 0.0008 \\
Hypercalcemia & 1.29 & $0.81-2.05$ & 0.29 \\
T $>5$ cm & 1.42 & $1.12-1.81$ & 0.0037 \\
T4b & 1.47 & $1.11-1.96$ & 0.0079 \\
N1 & 1.17 & $0.93-1.47$ & 0.19 \\
M1 & 1.83 & $1.48-2.27$ & $<0.0001$ \\
\hline
\end{tabular}

determined in accordance with the 7th edition of the Union for International Cancer Control (UICC) classification system. As shown in Table 1, age $\geq 70$ years, T4b tumor and all 4 factors for PI were confirmed to be significant risk factors for cause-specific death from ATC. CSS curves for each UICC stage are shown in Figure 2. MST and 6-month CSS rate were 236 days and $60 \%$ for stage IVA; 147 days and $45 \%$ for stage IVB and 81 days and $19 \%$ for stage IVC, respectively $(\mathrm{p}<.0001)$. When applying PI to the ATCCJ database, MST and 6-month CSS were also significantly different between groups ( $\mathrm{p}<.0001)$ : 274 days and $81 \%$ for $\mathrm{PI}=0 ; 226$ days and $61 \%$ for $\mathrm{PI}=1 ; 140$ days and $37 \%$ for $\mathrm{PI}=2$; 85 days and $17 \%$ for $\mathrm{PI}=3$; and 54 days and $11 \%$ for $\mathrm{PI}=4$.

In determining the appropriate treatment strategy for patients with ATC, consideration should be given to both UICC stage as an indication of disease extent and other prognostic factors representing the grade of biological malignancy. For patients who have few risk factors and can expect longer survival, intensive treatment is recommendable to pursue the best survival results. On the other hand, for patients who cannot anticipate good survival results from the analysis of prognostic factors, aggressive therapies are likely to markedly worsen QOL and even occasionally shorten survival. For such patients, it is justifiable to use the best supportive care and local disease control to maintain quality of survival and to prevent death from suffocation, dysphagia and bleeding, despite the lack of survival benefit.

Cause-specific survival (\%)

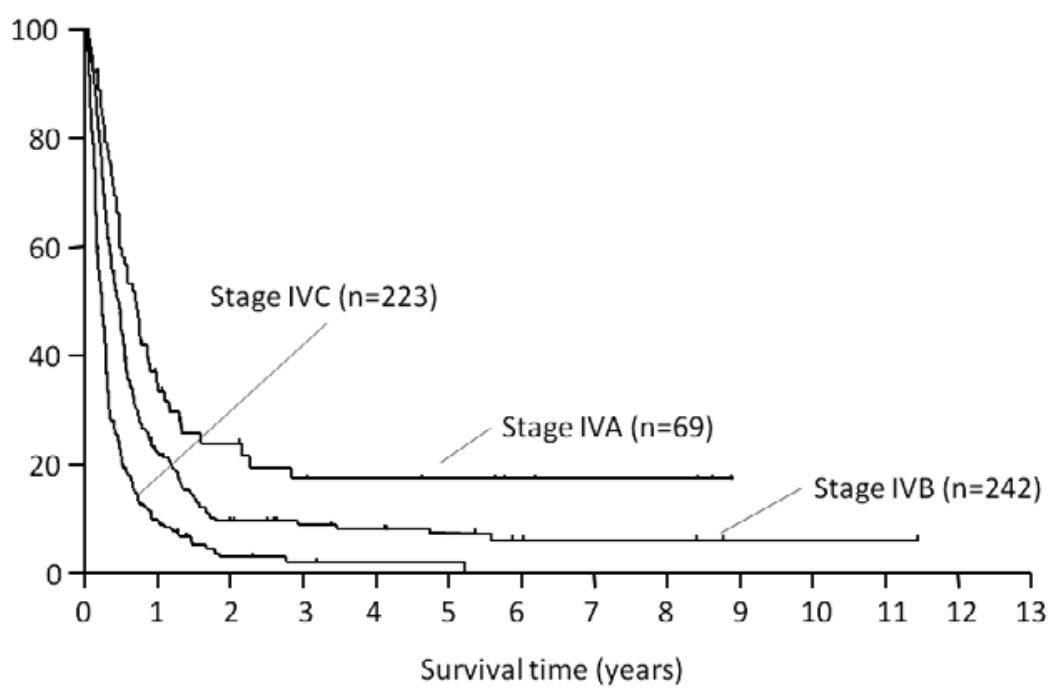

Fig. 2 Cause-specific survival curves for each UICC stage of anaplastic thyroid carcinoma 


\section{Effectiveness of Each Treatment Modality for Respective Stages of ATC}

In terms of multimodal treatment for ATC, adequate combinations and sequences of therapy for prolonged survival have not yet been sufficiently clarified ${ }^{6-9}$. As for the relationship between treatment methods and outcomes in patients with common-type ATC, radical surgery compared to no or palliative surgery (hazard ratio [HR]: 0.35; 95\% confidence interval [CI]: 0.28-0.43; p $<.0001), \geq 40$ Gy of RTX compared to no or $<40$ Gy RTX (HR: 0.46; 95\% CI: 0.38-0.56; $\mathrm{p}<.0001$ ) and any CTX compared with no CTX (HR: 0.63; 95\%CI: 0.52-0.76; p<.0001) were associated with significantly better outcomes.

We investigated the effects of adjuvant therapies including RTX and CTX on patients who underwent radical surgery. For stage IVA patients, although the benefit from additional therapies was not significant, patients who underwent RTX after radical surgery tended to show better (HR: 0.37; 95\% CI: 0.12-1.13; p=.081) outcomes than those who only underwent radical surgery. As for stage IVB patients who received radical surgery, adjuvant multimodal therapy with both RTX and CTX significantly improved CSS compared to radical surgery only or surgery with RTX (HR: 0.45 ; 95\% CI: $0.25-0.81$; p $=.0083)$.

\section{Super-radical Surgery on Stage IVB ATC}

Locally curative resection is considered to be the cornerstone for longer survival for patients with $\mathrm{ATC}^{10}$. Unfortunately, most ATCs infiltrate beyond the thyroid capsule and invade adjacent vital organs. Thus, complete resection of all gross disease without sacrificing vital structures can be performed only in a particular subset of patients with localized disease. The indication of superradical surgery including full-thickness resection of the trachea, larynx, and/or esophagus is controversial. Using the ATCCJ database, we carried out a retrospective study examining the efficacy of super-radical surgery on stage IVB common-type ATC ${ }^{11}$.

Here, super-radical surgery was defined as: (1) segmental or total resection of the trachea/larynx or esophagus/pharynx; (2) mediastinal operation with osteotomy; or (3) resection of the major cervical arteries. Resection of the recurrent laryngeal nerve, shaving of the tracheal wall or muscle layer resection of the esophagus were not included. Restricted radical surgery represented curative resection by means of thyroidectomy and cervical lymph node dissection, occasionally including resection of the muscles, veins, recurrent laryngeal nerve and/or superfi-

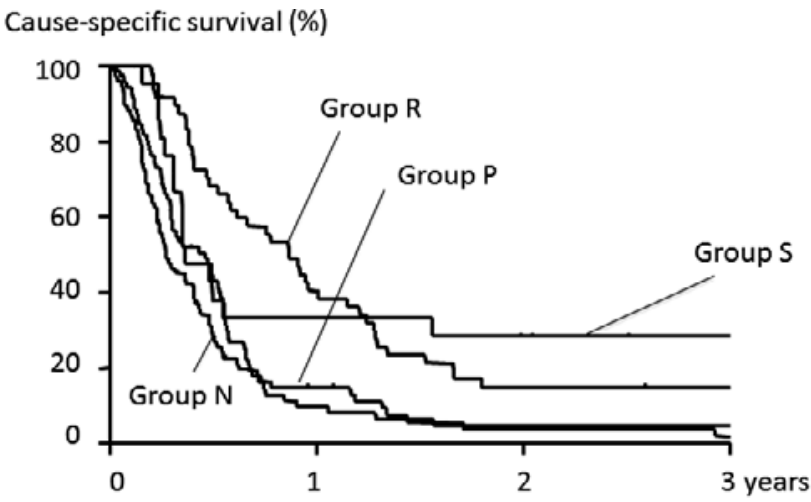

Fig. 3 Survival curves for each surgical group Group R: patients who underwent restricted radical surgery

Group S: patients who underwent super-radical surgery Group P: patients who underwent palliative surgery Group N: patients who underwent no surgery

cial shaving of the aerodigestive tract. Palliative resection denoted reduction surgery with macroscopically evident residual tumor. Among patients with common-type ATC, 233 patients were in stage IVB (43\%). Out of these, 23 patients underwent super-radical surgery (group S); 49 received restricted radical surgery (group R); 72 had palliative surgery (group P) and 80 patients got no surgery (group N). Procedures of super-radical surgeries were total pharyngo-laryngectomy in 12; total laryngectomy in 6; tracheal resection in 3; esophageal resection in 1 and mediastinal operation in 3 ( 2 of them were combined with other procedures).

Treatment outcomes are shown in Figure 3. MST and 1-year CSS were 315 days and 41\% for group R; 129 days and 33\% for group S; 136 days and 15\% for group P; 101 days and $10 \%$ for group $\mathrm{N}$, respectively. Patients in group $\mathrm{R}$ displayed the best survival outcomes and the difference between group $\mathrm{R}$ and groups $\mathrm{P}$ or $\mathrm{N}$ was significant $(\mathrm{p}<.0001)$. Patients in group $\mathrm{S}$ also showed significantly better survival than those in groups $\mathrm{P}(\mathrm{p}=.03)$. Survival rates in groups $\mathrm{R}$ and $\mathrm{S}$ were almost identical ( $\mathrm{p}$ $=.94)$. Among group S, 7 (30\%) patients survived more than 1 year. For PI $\leq 1$ patients, 1-year CSS was 50\%; whereas for $\mathrm{PI} \geq 2$, it was $11 \%(\mathrm{p}=.15)$. Permanent tracheostomy was significantly more frequent in group $\mathrm{S}$ (18 out of $23: 78 \%$ ) than in other groups $(\mathrm{p}<.0001)$. After the curative surgery, $33(67 \%)$ patients in group $\mathrm{R}$ received $\geq 40$ Gy of RTX and/or any kind of CTX; however, only $9(39 \%)$ patients in group $S$ had the adjuvant therapies. Two patients in group $S$ died from surgical complications.

Aggressive surgery offers significantly better outcomes 
Table 2 Comparison of clinico-pathological characteristics between incidental ATC and common-type ATC

\begin{tabular}{lccc}
\hline & $\begin{array}{c}\text { Incidental } \\
\text { ATC }\end{array}$ & $\begin{array}{c}\text { Common-type } \\
\text { ATC }\end{array}$ & $\mathrm{p}$ \\
\hline Age (years) & $67 \pm 11$ & $69 \pm 11$ & 0.734 \\
Sex (Male : Female) & $3: 22(1: 7.3)$ & $208: 338(1: 1.6)$ & 0.010 \\
Tumor size (cm) & $2.1 \pm 2.1$ & $6.5 \pm 2.6$ & 0.000 \\
Extrathyroidal invasion & $13(52 \%)$ & $426(80 \%)$ & 0.004 \\
Distant metastasis & $3(13 \%)$ & $215(41 \%)$ & 0.005 \\
Co-existing tumor (PTC : FT) & $24: 1$ & $123: 28(4.3: 1)$ & 0.000 \\
\hline
\end{tabular}

PTC: papillary thyroid carcinoma, FT: follicular tumor

than limited surgery for patients with stage IVB ATC. Super-radical procedures may be indicated in selected patients like PI $\leq 1$ and can sometimes achieve fairly long survival. A tragic end due to locally uncontrolled disease can be avoided at the expense of diminished quality of life, including loss of voice. Surgical indications for ATC should be considered from the perspective of surgical skill, biological characteristics of the tumor, general condition of patients, and the intentions of the patient and their family.

\section{Incidental ATC}

In surgery for patients with preoperative diagnosis of DTC, ATC is occasionally detected incidentally on postoperative pathological examination. Here, we defined incidental ATC as largely DTC accompanied by a minute $(1-2 \mathrm{~cm})$ region of ATC and reviewed the detailed information about 25 cases of incidental ATC from the ATCCJ database ${ }^{12}$.

The clinico-pathological characteristics of incidental ATC were compared to common-type ATC (Table 2). There was no difference in age between the 2 groups; but the percentage of females was significantly higher in those with incidental ATC. The tumor size was smaller, and patients with extrathyroidal invasion or distant metastasis were significantly fewer in the incidental ATC group than in the common-type ATC group. Most incidental ATCs coexisted with papillary thyroid carcinoma (PTC). They may represent an initial stage of anaplastic transformation from PTC to ATC.

As shown in Figure 1, patients with incidental ATC showed significantly better survival than patients with common-type ATC $(\mathrm{p}<.001)$. One-year and 2-year CSS rate were $72 \%$ and $58 \%$, respectively; however, nearly half (12 out of $25 ; 48 \%$ ) of the patients died of the disease eventually.

Regarding treatment modalities for patients with inci- dental ATC, the outcome was better in those who underwent curative resection ( $\mathrm{n}=19$; 1-year CSS: 84\%) than in those without ( $\mathrm{n}=6$; 1-year CSS: 33\%; $\mathrm{p}=.001$ ). Additional use of RTX and/or CTX after curative resection could slightly improve survival, but it did not reach statistical significance (HR: 0.28; 95\% CI: 0.08-1.03; $\mathrm{p}=0.055$ ).

Incidental ATC would be the only curable ATC, and postoperative adjuvant RTX and/or CTX is recommended to achieve long-term survival. Further studies are needed to establish the contents and details of additional therapies for patients with incidental ATC.

\section{Histopathological Features of ATC with Long-term Survival}

The pathological advisory team of the ATCCJ investigated the histopathological features of ATC in patients who achieved long-term survival ${ }^{13}$. From the ATCCJ database, 88 ATC cases in which the patient died within 3 months (short-term survival; STS) and 68 ATC cases in which the patient survived longer than 1 year (long-term survival; LTS) were corrected. Three thyroid-specialized pathologists reviewed the specimen from 12 institutions.

Six $(7 \%)$ STS cases and $27(40 \%)$ LTS cases were considered not to be ATC after the central review. Of these, $22(67 \%)$ were reclassified as PTC. They included PTC with squamous cell carcinoma (12 cases), PTC with squamous metaplasia (3 cases), PTC with a focal insular component ( 2 cases), a columnar cell variant (2 cases), and a diffuse sclerosing variant (1 case). The other cases included poorly differentiated carcinoma, carcinoma showing thymus-like differentiation (CASTLE), squamous cell carcinoma (SCC), mucoepidermoid carcinoma, malignant lymphoma, metastatic carcinoma and atypical adenoma. The differential diagnosis of ATC is very broad, and careful examination is needed before making a diagnosis, especially for cases with SCC components. The central review board of expert pathologists should play 
an important role in establishing an accurate diagnosis of ATC, particularly if the patient had longer survival.

In cases without CTX, LTS was significantly more common if there was a pre-existing tumor (DTC), epithelial growth, or lymphocytic infiltration, and STS was more common if neutrophilic infiltration was present. In cases with CTX, LTS was significantly more common if epithelial growth or a SCC component was present, whereas STS was more common in cases with rhabdoid cells. The presence of a pre-existing tumor, epithelial growth, a SCC component, no neutrophilic infiltration and lymphocytic infiltration may be favorable prognostic factors in ATC. This is the first report to identify microscopic features related to the prognosis of ATC in a large series. Immunohistochemical results including cytokeratin AE1/ AE3, TTF-1, p63, p53 and Ki-67 labeling index were not related to survival.

\section{Investigator-initiated, Nationwide Clinical Trials Conducted by ATCCJ}

(1) The Feasibility, Safety and Efficacy Study of Weekly Paclitaxel Administration for Patients with ATC (Attack J Study, UMIN: 000008574)

Systemic CTX is mandatory for most ATC patients; however, conventional chemotherapeutic treatment using doxorubicin or cisplatin did not demonstrate any significant effect. More recent studies have suggested a potential benefit of taxans to control advanced disease ${ }^{14,15}$. Higashiyama et al. $^{16}$ reported their retrospective experience of induction CTX with a weekly paclitaxel administration in 13 patients (9: stage IVB; 4: stage IVC): a response rate of $31 \%$ with 1 complete remission (CR) was observed. Overall survival of stage IVB patients with induction CTX was better $(p=.0213)$ than that without the CTX and also better $(p=.0467)$ than those with CTX other than paclitaxel. They concluded that weekly administration of paclitaxel could be beneficial to extend the survival of ATC patients with stage IVB disease.

The ATCCJ have conducted an investigator-initiated prospective clinical trial to objectively evaluate outcomes of treatment with paclitaxel since April 2012 ${ }^{17,18}$. A multicenter, nonrandomized, open-label, single-arm study to evaluate the feasibility and efficacy of weekly paclitaxel $\left(80 \mathrm{mg} / \mathrm{m}^{2}\right)$ administration for patients with ATC was carried out by a nationwide organization. Histopathological diagnosis was confirmed by central review. By February 2014, 71 patients were registered from 28 institutions and followed until March, 2015. Feasibility was analyzed in 56 patients. More than 1 course of treatment
(3 weeks) was performed in 52 (93\%) patients retaining sufficient dose intensity ( $>84 \%)$. All the adverse events (AEs) observed in the study were tolerable and manageable. No patients had to terminate the treatment because of an adverse event.

The MST was 6.7 months (95\% CI: 4.4-9.0). The 6month CSS was 54\%. Among the 42 patients with an evaluable lesion, none demonstrated CR, 9 (21\%) showed partial remission (PR), 22 (52\%) achieved stable disease (SD), and 8 (19\%) exhibited progressive disease (PD); 3 did not complete the initial treatment course. The objective response rate (ORR: $\mathrm{CR}+\mathrm{PR}$ ) was $21 \%$, and the clinical benefit rate $(\mathrm{CR}+\mathrm{PR}+\mathrm{SD})$ was $73 \%$. The median time to progression was 1.6 months. Statistically, no additional effect of concomitant radiation was demonstrated in 6 patients receiving combined therapy. Eight patients, in whom a complete post-treatment surgical removal of the tumor was feasible, survived significantly longer (MST: 7.6 months; 95\% CI: 8.1-23.0) than the other 34 patients in whom the tumor could not be completely removed after CTX (MST: 5.4 months; 95\% CI: 3.0-7.8; p=.019).

The study demonstrates objective and accurate information concerning the feasibility and efficacy of a standardized treatment with weekly paclitaxel administration for ATC patients. Weekly paclitaxel administration for ATC patients might be of clinical benefit in a neoadjuvant setting. In addition, this protocol could be safely conducted in an outpatient setting.

(2) Phase II Study Assessing the Efficacy and Safety of Lenvatinib for ATC (HOPE Study, UMIN: 000020773)

Recently, phase III clinical trials demonstrated that multi-targeted tyrosine kinase inhibitors (TKIs) had clinical efficacy to extend progression-free survival (PFS) significantly in advanced or metastatic radioactive-iodine refractory DTC ${ }^{19,20}$. In Japan, a single-arm, open-label, phase II study of lenvatinib in patients with DTC, medullary thyroid carcinoma and ATC (NCT01728623) was conducted from 2012. Initial report in 2014 showed 5 of 11 patients with ATC have received $>6$ months of treatment with lenvatinib and 3 patients showed $\mathrm{PR}^{21}$. Finally, 17 patients with ATC were enrolled. The median PFS was 7.4 months (95\% CI: 1.7-12.9), the median overall survival (OS) was 10.6 months (95\% CI: 3.8-19.8), and the ORR was $24 \%$ (4 out of 17$)^{22}$. In 2015, lenvatinib was eventually approved by the regulatory agency for clinical use for the treatment of thyroid cancer, including ATC in Japan. This promising medicine has a variety of AEs such as hypertension, fatigue, loss of appetite, and proteinuria, which are different from conventional cytotoxic 


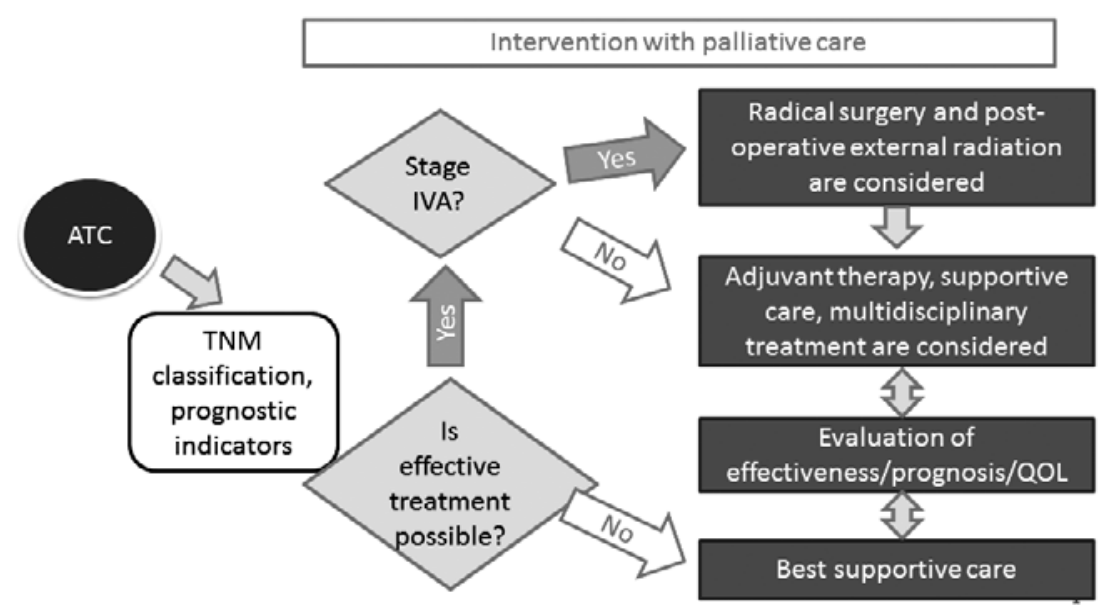

Fig. 4 Algorithm for diagnosis and treatment of anaplastic thyroid carcinoma in "Japanese guidelines for treatment of thyroid tumor 2010" (revised from reference \#24)

QOL: quality of life

agents. Although most of them were tolerable and manageable by dose adjustments; some AEs, such as massive bleeding form major vessels or fistula formation between important organs, may cause immediate life-threatening situations and major defects in the QOL of the patients ${ }^{23}$. Moreover, these TKIs inhibiting vascular endothelial growth factor (VEGF) also have a function to interfere with wound healing. It is crucial to select appropriate patients and determine the proper timing to start the therapy. Further investigation is needed to clarify the clinical efficacy and safety of lenvatinib in the treatment of ATC.

Thus, the ATCCJ has started another investigatorinitiated, multi-center, phase II study assessing the efficacy (primary endpoint: OS) and safety of lenvatinib for ATC since January 2016. Patients with unresectable ATC which is histologically confirmed at central review have been recruited.

\section{Revised Japanese Guidelines for Treatment of ATC Based on Upgraded Evidence}

"Japanese guidelines for treatment of thyroid tumor", which was edited by Japan Association of Endocrine Surgeons (JAES) and Japanese Society of Thyroid Surgery (JSTS), was published in 2010. The guidelines for ATC stated that it was not possible to present scientific evidence that would be a guide to treatment, because there had been few randomized or even prospective trials at that time. They proposed a simple algorithm for treatment of ATC (Fig. 4) ${ }^{24}$.

Revised Japanese guidelines are set to be published in 2017. The algorithm will be reformed adopting some of the ATCCJ's accomplishments (Fig. 5). For example, it will note individual therapeutic strategies for patients with ATC considering both the prognostic index and UICC staging, to differentiate cases that could benefit from an effort to prolong life and cases that should be switched to best supportive care. The ATCCJ is now planning to conduct several clinical trials to fill in blanks of the algorithm and establish standardized therapeutic strategy for ATC as follows: (1) phase II study of neoadjuvant CTX with weekly-paclitaxel for resectable ATC; (2) phase III study of adjuvant therapy after curative surgery (weekly-paclitaxel vs. weekly-paclitaxel+RTX); and (3) phase I \& II studies to clarify the feasibility and efficacy of palliative chemo-radiotherapy.

\section{Summary}

After a long, dark outlook with this formidable disease, there are glimmers of hope for progress. A nationwide multi-institutional collaborative study group is strengthening the activity to recruit orphan disease patients. Big clinical data accompanied with a clinical trial network may help to uncover new strategies to conquer this dismal malignancy in the near future.

Acknowledgements: On behalf of the ATCCJ, the authors acknowledge and thank the following doctors and participating centers for their involvement in this nationwide study: Dr. Hideo Kurihara, Department of Surgery, Kurihara Clinic; Dr. Yuki Tomisawa, Department of Surgery, Uchimaru Hospital; Dr. Satoshi Ogasawara, Department of Surgery, Koujin Hospital; Dr. Michio Watanabe, Department of Breast and Endocrine Surgery, Iwate Prefectural Central Hospital; Dr. Kiyoto Shiga, Department of Otolaryngology-Head and Neck Surgery, Iwate 


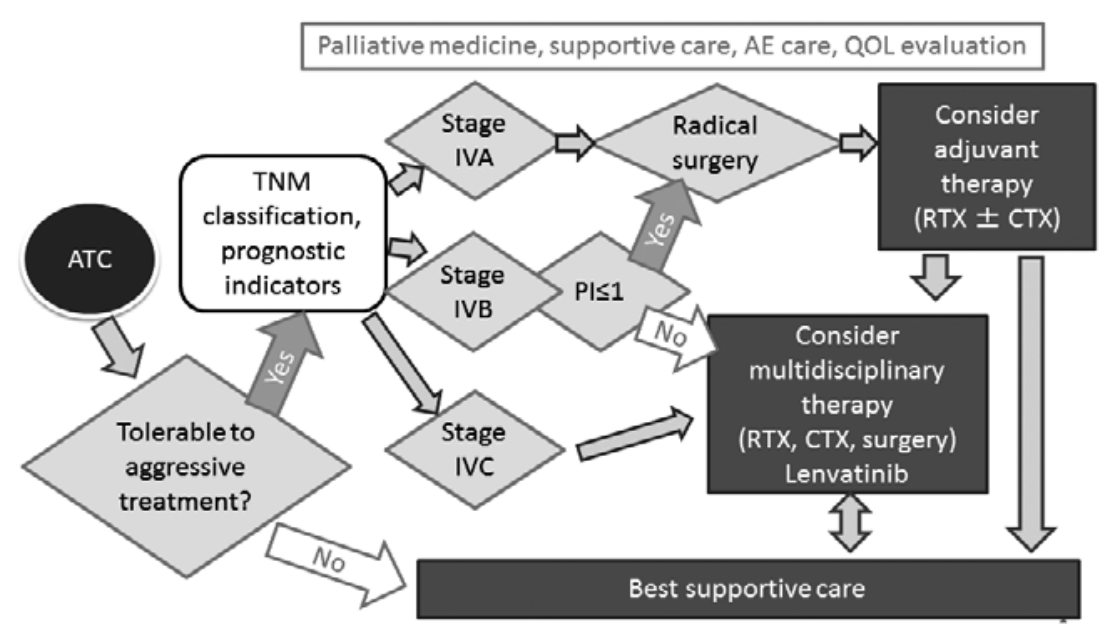

Fig. 5 Algorithm for diagnosis and treatment of anaplastic thyroid carcinoma in revised Japanese guidelines for treatment of thyroid tumor 2017 (draft)

PI: prognostic index

RTX: external beam radiotherapy

CTX: chemotherapy

AE: adverse event

QOL: quality of life

Medical University; Dr. Akihiro Homma, Department of Otolaryngology-Head and Neck Surgery, Hokkaido University Graduate School of Medicine; Dr. Tomohiro Sakashita, Department of Otolaryngology-Head and Neck Surgery, Kushiro City General Hospital; Dr. Atsushi Fukuuchi, Department of Breast and Endocrine Surgery, Mitsui Memorial Hospital; Dr. Seiichi Yoshimoto, Department of Head and Neck Oncology, National Cancer Center Hospital; Dr. Koki Miura, Head and Neck Oncology Center, International University of Health and Welfare, Mita Hospital; Dr. Takayoshi Niwa, Department of Breast and Endocrine Surgery, University of Tokyo; Dr. Tatsuya Fukumori, Department of Surgery, Kanaji Thyroid Hospital; Dr. Kazuhisa Toda, Division of Head and Neck, Cancer Institute Hospital; Dr. Kiminori Sugino, Department of Surgery, Ito Hospital; Dr. Hidemitsu Tsutsui, Department of Respiratory and Thyroid Surgery, Tokyo Medical University; Dr. Takahiro Okamoto, Department of Endocrine Surgery, Tokyo Women's Medical University; Dr. Kiyoaki Tsukahara, Department of Otolaryngology, Department of Otorhinolaryngology, Head and Neck Surgery, Tokyo Medical University; Dr. Tsuguo Iwatani, Division of Breast and Endocrine Surgery, Department of Surgery, St. Marianna University School of Medicine; Dr. Yoshiyuki Kadokura, Department of Otolaryngology, Showa University Northern Yokohama Hospital; Dr. Nobuhiro Fukunari, Department of Surgery, Showa University School of Medicine, Yokohama Northern Hospital; Dr. Katsuhiko Masudo, Department of Breast and Thyroid Surgery, Yokohama City University Medical Center; Dr. Hirotaka Nakayama, Department of Surgery, Yokohama City University; Dr. Ryo Ikoma, Department of Otolaryngology, Yoko- hama Minami Kyousai Hospital; Dr. Hiroyuki Iwasaki, Department of Breast and Endocrine Surgery, Kanagawa Cancer Center; Dr. Hiroshi Katoh, Department of Surgery, Kitasato University School of Medicine; Dr. Makoto Kammori, Department of Breast and Thyroid surgery, Ichikawa Hospital, International University of Health and Welfare; Dr. Susumu Okano, Department of Head and Neck Oncology, National Cancer Center East; Dr. Toshihumi Tomioka, Department of Head and Neck Surgery, National Cancer Center East; Dr. Toshimitsu Nemoto, Department of Otolaryngology, Japanese Red Cross Narita Hospital; Dr. Minoru Fujimori, Department of Breast Surgery, Tokyo Medical University, Ibaraki Medical Center; Dr. Hisato Hara, Department of Breast and Endocrine Surgery, University of Tsukuba; Dr. Masashi Sugasawa, Department of Head and Neck Oncology, Saitama Medical University International Medical Center; Dr. Daiki Uchida, Toyohashi Medical Center, Dr. Kazuki Yokoi, Department of Surgery, Okazaki City Hospital; Dr. Yasuhisa Hasegawa, Department of Head and Neck Surgery, Aichi Cancer Center; Dr. Tsuneo Imai, National Hospital Organization, Higashinagoya National Hospital; Dr. Toyone Kikumori, Department of Breast and Endocrine Surgery, Nagoya University; Dr. Chikara Kagawa, Department of Endocrine Surgery, Fujita Health University; Dr. Shogo Nakano, Division of Breast and Endocrine Surgery, Department of Surgery, Aichi Medical University; Dr. Hiroyuki Yamada, Department of Otolaryngology, Ise RedCross Hospital; Dr. Masayuki Tori, Department of Surgery, Osaka Police Hospital; Dr. Atsuhiko Uno, Department of Otolaryngology, Osaka General Medical Center; Dr. Morimasa Kitamura, Department of Otolaryngology, Head and Neck Sur- 
gery, Graduate School of Medicine, Kyoto University; Dr. Katsunari Yane, Department of Otorhinolaryngology, Nara Hospital, Kinki University School of Medicine; Dr. Ichiro Ota, Department of Otorhinolaryngology-Head and Neck Surgery, Nara Medical University; Dr. Muneki Hotomi, Department of Otolaryngology-Head and Neck Surgery, Wakayama Medical University; Dr. Takuya Higashiyama, Department of Surgery, Kuma Hospital; Dr. Naomi Kiyota, Department of Medical Oncology and Hematology, Kobe University Hospital; Dr. Hiroya Kitano, Division of Otolaryngology-Head and Neck Surgery, Department of Medicine of Sensory and Motor Organs, School of Medicine, Faculty of Medicine Tottori University; Dr. Yorihisa Orita, Department of Otolaryngology, Okayama Saiseikai General Hospital; Dr. Katsuhiro Tanaka, Department of Breast and Thyroid Surgery, Kawasaki Medical School; Dr. Keizo Sugino, Department of Surgery, Akane Foundation Tsuchiya General Hospital; Dr. Nobuya Monden, Department of Head and Neck Surgery, Shikoku Cancer Center; Dr. Hiroyuki Yamashita, Yamashita Thyroid \& Parathyroid Clinic; Dr. Kosho Yamanouchi, Department of Surgery, Nagasaki University; Dr. Shigeto Maeda, Department of Surgery, National Nagasaki Medical Center; Dr. Shiro Noguchi, Department of Surgery, Noguchi Thyroid Clinic and Hospital Foundation; Dr. Takao Miyaguni, Department of Surgery, Naha City Hospital; Dr. Yuichiro Sato, Division of Head and Neck Surgery, Niigata Cancer Center Hospital; Dr. Hirohumi Ami, Department of Surgery, Ohara General Hospital; Dr. Tsuyoshi Yoshida, Department of Otolaryngology, Takeda General Hospital; Dr. Keisei Fujimori, Department of Breast and Endocrine Surgery, Tohoku University Hospital; and Dr. Tomoko Yamazaki, Department of Head and Neck Oncology, Miyagi Cancer Center.

In addition, we sincerely thank the following doctors for giving scientific advice to our studies: Dr. Atsuhiko Sakamoto, Dr. Kenichi Kakudo, Dr. Mitsuyosi Hirokawa, Dr. Akira Miyauchi, Dr. Akira Yoshida, Dr. Shunichi Yamashita, Dr. Norisato Mitsutake, Dr. Hiroshi Takami and Dr. Yoshihide Fujimoto.

Conflict of Interest: IS has research grant from Eisai, fees for consulting and honoraria from Eisai, Bayer and Sanofi. NO has grant from Osaka City University (Novel Priority Research B) and JSPS (KAKENHI \#25461992). NO also has research grant from Eisai and Bayer, and is a member of advisory board for Eisai and Bayer. NO has honoraria from Eisai, Bayer, Aska pharma, MDS, Covidien, Johnson \& Johnson and Cosmic cooperation. Novartis provided drugs for research conducted by NO.

\section{References}

1. Hundahl SA, Fleming ID, Fremgen AM, Menck HR: A National Cancer Database report on 53,856 cases of thy- roid carcinoma treated in the U.S., 1985-1995. Cancer 1998; 83: 2638-2648.

2. Kitamura $Y$, Shimizu $K$, Nagahama $M$, Sugino $K$, Ozaki $\mathrm{O}$, Mimura T, Ito K, Tanaka S: Immediate causes of death in thyroid carcinoma: clinicopathological analysis of 161 fatal cases. J Clin Endocrinol Metab 1999; 84: 4043-4049.

3. Sugitani I, Miyauchi A, Sugino K, Okamoto T, Yoshida A, Suzuki S: Prognostic factors and treatment outcomes for anaplastic thyroid carcinoma: ATC research consortium of Japan cohort study of 677 patients. World J Surg 2012; 36: 1247-51254.

4. Sugitani I, Kasai N, Fujimoto Y, Yanagisawa A: Prognostic factors and therapeutic strategy for anaplastic carcinoma of the thyroid. World J Surg 2001; 25: 617-622.

5. Orita Y, Sugitani I, Amemiya T, Fujimoto Y: Prospective application of our prognostic index in the treatment of anaplastic thyroid carcinoma. Surgery 2011; 150: 12121219.

6. Haigh PI, Ituarte PH, Wu HS, Treseler PA, Posner MD, Quivey JM, Duh QY, Clark OH: Completely resected anaplastic thyroid carcinoma combined with adjuvant chemotherapy and irradiation is associated with prolonged survival. Cancer 2001; 91: 2335-2342.

7. Chen J, Tward JD, Shrieve DC, Hitchcock YJ: Surgery and radiotherapy improves survival in patients with anaplastic thyroid carcinoma: analysis of the surveillance, epidemiology, and end results 1983-2002. Am J Clin Oncol 2008; 31: 460-464.

8. Swaak-Kragten AT, de Wilt JHW, Schmitz PIM, Bontenbal M, Levendag PC: Multimodality treatment for anaplastic thyroid carcinoma: treatment outcome in 75 patients. Radiother Oncol 2009; 92: 100-104.

9. Ito $\mathrm{K}$, Hanamura T, Murayama $\mathrm{K}$, Okada $\mathrm{T}$, Watanabe $\mathrm{T}$, Harada M, Ito T, Koyama H, Kanai T, Maeno K, Mochizuki Y, Amano J: Multimodality therapeutic outcomes in anaplastic thyroid carcinoma: improved survival in subgroups of patients with localized primary tumors. Head Neck 2012; 34: 230-237.

10. Ito $Y$, Higashiyama $T$, Hirokawa $M$, Fukushima M, Inoue H, Yabuta T, Tomoda C, Uruno T, Kihara M, Takamura Y, Miya A, Kobayashi K, Matsuzuka F, Miyauchi A: Investigation of the validity of UICC stage grouping of anaplastic carcinoma of the thyroid. Asian J Surg 2009; 32: 47-50.

11. Sugitani I, Hasegawa $Y$, Sugasawa M, Tori M, Higashiyama T, Miyazaki M, Hosoi H, Orita Y, Kitano H: Superradical surgery for anaplastic thyroid carcinoma: a large cohort study using the anaplastic thyroid carcinoma research consortium of Japan database. Head Neck 2014; 36: 328-333.

12. Yoshida A, Sugino K, Sugitani I, Miyauchi A: Anaplastic thyroid carcinomas incidentally found on postoperative pathological examination. World J Surg 2014; 38: 23112316.

13. Hirokawa M, Sugitani I, Kakudo K, Sakamoto A, Higashiyama T, Sugino K, Toda K, Ogasawara S, Yoshimoto S, Hasegawa Y, Imai T, Onoda N, Orita Y, Kammori M, Fujimori K, Yamada H: Histopathological analysis of anaplastic thyroid carcinoma cases with long-term survival: a report from the Anaplastic Thyroid Carcinoma Research Consortium of Japan. Endocr J 2016; 63: 441447.

14. Ain KB, Egorin MJ, DeSimone PA: Treatment of anaplastic thyroid carcinoma with paclitaxel: phase 2 trial using ninety-six-hour infusion. Collaborative Anaplastic Thyroid Cancer Health Intervention Trials (CATCHIT) Group. Thyroid 2000; 10: 587-594. 
15. Kawada K, Kitagawa K, Kamei S, Inada M, Mitsuma A, Sawaki M, Kikumori T, Fujimoto Y, Arima H, Imai T, Ando Y: The feasibility study of docetaxel in patients with anaplastic thyroid cancer. Jpn J Clin Oncol 2010; 40: 596-599.

16. Higashiyama T, Ito $\mathrm{Y}$, Hirokawa $\mathrm{M}$, Fukushima M, Uruno T, Miya A, Matsuzuka F, Miyauchi A: Induction chemotherapy with weekly paclitaxel administration for anaplastic thyroid carcinoma. Thyroid 2010; 20: 7-14.

17. Onoda N, Sugitani I, Higashiyama T, Hara H, Ito K, Kammori M, Sugino K, Suzuki S, Toda K, Yoshida A, Miyauchi A: Concept and design of a nationwide prospective feasibility/efficacy/safety study of weekly paclitaxel for patients with pathologically confirmed anaplastic thyroid cancer (ATCCJ-PTX-2). BMC Cancer 2015; 20: 475.

18. Onoda N, Sugino K, Higashiyama T, Kammori M, Toda K, Ito K, Yoshida A, Suganuma N, Nakashima N, Suzuki S, Tsukahara K, Noguchi H, Koizumi M, Nemoto T, Hara $\mathrm{H}$, Miyauchi A, Sugitani I: The safety and efficacy of weekly paclitaxel administration for anaplastic thyroid cancer patients: a nationwide prospective study. Thyroid 2016; 26: 1293-1299.

19. Brose MS, Nutting CM, Jarzab B, Elisei R, Siena $S$, Bastholt L, de la, Fouchardiere C, Pacini F, Paschke R, Shong YK, Sherman SI, Smit JW, Chung J, Kappeler C, Peña C, Molnár I, Schlumberger MJ; DECISION investigators: Sorafenib in radioactive iodine-refractory, locally advanced or metastatic differentiated thyroid cancer: a randomized, double-blind, phase 3 trial. Lancet 2014; 384: 319-328.

20. Schlumberger M, Tahara M, Wirth LJ, Robinson B, Brose
MS, Elisei R, Habra MA, Newbold K, Shah MH, Hoff AO, Gianoukakis AG, Kiyota N, Taylor MH, Kim SB, Krzyzanowska MK, Dutcus CE, de las Heras B, Zhu J, Sherman SI: Lenvatinib versus placebo in radioiodinerefractory thyroid cancer. N Eng J Med 2015; 372: 621630.

21. Takahashi S, Tahara M, Kiyota N, Yamazaki T, Chayahara N, Nakano K, Inagaki L, Toda K, Enokida T, Minami H, Imamura Y, Sasaki T, Suzuki T, Fujino K, Dutcus CE: Phase II study of lenvatinib, a multi-targeted tyrosine kinase inhibitor, in patients with all histologic subtypes od advanced thyroid cancer. Ann Oncol 25 2014; Abstract 4933 (supple 4): iv340-iv356.

22. Tahara M, Kiyota N, Yamazaki T, Chayahara N, Nakano K, Inagaki L, Toda K, Enokida T, Minami H, Imamura Y, Sasaki T, Suzuki T, Fujino K, Dutcus CE, Takahashi S: Lenvatinib for anaplastic thyroid cancer. Front Oncol 2017; 7: 25 .

23. Obata K, Sugitani I, Ebina A, Sugiura Y, Toda K, Takahashi S, Kawabata K: Common carotid artery rupture during treatment with lenvatinib for anaplastic thyroid cancer. Int Canc Conf J 2016; doi 10.1007/s13691-0160257-7.

24. Sugino K, Onoda N: Anaplastic thyroid carcinoma in Treatment of Thyroid Tumor, Japanese Clinical Guidelines (Takami H, Ito Y, Noguchi H, Yoshida A, Okamoto T, eds), 2010; 11, 203-227, Springer, Tokyo.

(Received, August 29, 2017)

(Accepted, November 16, 2017) 